\title{
Review
}

\section{T-cell Responses to Dengue Virus in Humans}

\author{
Ichiro Kurane ${ }^{1 *}$, Takaji Matsutani ${ }^{2}$, Ryuji Suzuki ${ }^{3}$, Tomohiko Takasaki ${ }^{4}$, Siripen Kalayanarooj ${ }^{5}$, \\ Sharone Green ${ }^{6}$, Alan L. Rothman ${ }^{6,7}$ and Francis A. Ennis ${ }^{6}$ \\ Published online 1 December, 2011
}

\begin{abstract}
Dengue virus (DENV) is a leading cause of morbidity and mortality in most tropical and subtropical areas of the world. Dengue virus infection induces specific CD4+CD8- and CD8+CD4- T cells in humans. In primary infection, T-cell responses to DENV are serotype cross-reactive, but the highest response is to the serotype that caused the infection. The epitopes recognized by DENV-specific T cells are located in most of the structural and non-structural proteins, but NS3 is the protein that is most dominantly recognized. In patients with dengue hemorrhagic fever (DHF) caused by secondary DENV infection, T cells are highly activated in vivo. These highly activated T cells are DENV-specific and oligoclonal. Multiple kinds of lymphokines are produced by the activated $\mathrm{T}$ cells, and it has been hypothesized that these lymphokines are responsible for induction of plasma leakage, one of the most characteristic features of DHF. Thus, T-cells play important roles in the pathogenesis of DHF and in the recovery from DENV infection.
\end{abstract}

Key words: Dengue virus, T-cell response, T-cell receptor, Dengue fever, Dengue hemorrhagic fever

\section{INTRODUCTION}

Dengue fever (DF)/dengue hemorrhagic fever (DHF) is one of the most important infectious diseases in tropical and subtropical areas of the world. The areas where DF/DHF is a serious health concern have been expanding, and the number of DHF patients has been increasing. The immune responses to DENV have been analyzed in humans. The antibody responses are well known and the roles of specific antibodies in the prevention of DENV infection and in the pathogenesis of DHF have been established. On the other hand, T-cell responses to DENV is not as well understood as that of antibodies.

\section{DENGUE VIRUS (DENV)}

DENV belongs to the family Flaviviridae, the genus Flavivirus. The genome of dengue virus is a single, plus- stranded RNA nearly $11 \mathrm{~Kb}$ in length. The open reading frame codes three structural proteins (capsid (C) protein, preM protein, and envelope (E) protein) and seven nonstructural proteins (NS1, NS2a, NS2b, NS3, NS4a, NS4b and NS5). Dengue virion has a spherical shape and measures $40-50 \mathrm{~nm}$ in diameter. The envelope is a lipid bilayer containing two envelope-associated proteins: the $\mathrm{E}$ and $\mathrm{M}$ proteins.

DENV is transmitted to humans by infected mosquitoes, mainly Aedes aegypti and Aedes albopictus. Humans are natural hosts of DENV, and DENV is maintained between mosquitoes and humans in nature. There are four serotypes: DENV types 1, 2, 3 and 4. Although DENV types 1, 2, 3 and 4 are referred to as serotypes, it is generally accepted that the four serotypes are different albeit antigenically related species.

\footnotetext{
${ }^{1}$ National Institute of Infectious Diseases, Japan

${ }^{2}$ Laboratory of Immune Regulation, Wakayama Medical University, Japan

${ }^{3}$ Department of Rheumatology and Clinical Immunology, Clinical Research Center for Allergy and Rheumatology, Sagamihara National Hospital, Japan

${ }^{4}$ Department of Virology 1, National Institute of Infectious Diseases, Japan

${ }^{5}$ Dengue Unit, Queen Sirikit National Institute of Child Health, Bangkok, Thailand

${ }^{6}$ Department of Medicine, University of Massachusetts Medical School, U.S.A.

${ }^{7}$ Current address: Institute for Immunology and Informatics, University of Rhode Island, U.S.A.

*Corresponding author:

National Institute of Infectious Diseases, 1-23-1 Toyama, Shinjuku-ku, Tokyo 162-8640, Japan

Tel: $+81-3-5285-1111$

Fax: +81-3-5285-1356

E-mail: kurane@nih.go.jp
} 


\section{DENGUE FEVER (DF) AND DENGUE HEMORRHAGIC FEVER (DHF); TWO CLINICAL MANIFESTATIONS OF DENGUE VIRUS INFECTION}

Dengue virus infection can be asymptomatic or cause two forms of illness, DF and DHF, although the majority of DENV infections are asymptomatic. New diagnostic names have recently been proposed [1], but the terms DF and DHF are still widely used.

DF is a self-limiting febrile illness. After an incubation period of 2-7 days, a sudden onset of fever occurs. The fever is usually accompanied by retro-orbital or frontal headache. Myalgia and bone pain occur soon after the onset of fever. A transient macular rash that blanches under pressure, nausea, vomiting, lymphadenopathy and taste aberrations can develop. These symptoms are accompanied by leucopenia and variable degrees of thrombocytopenia. One to two days after defervescence, a generalized morbilliform maculopapular rash appears. The rash spares palms and soles. Patients usually recover from the symptoms without complications about a week after the onset of disease.

Some patients infected with dengue virus demonstrate plasma leakage into interstitial spaces, thrombocytopenia and also hemorrhagic manifestation. This severe life-threatening syndrome is called DHF. The incubation period of DHF is similar to that of DF. Illness starts with fever, malaise, vomiting, headache, anorexia, and cough. Rapid clinical deterioration and collapse follow after 2-5 days. In the second phase, the patients demonstrate cold clammy extremities, warm trunk, flushed face, restlessness, irritability, middle gastric pain, and may progress to a rapid weak pulse, hypotention and narrow pulse pressure. The crisis lasts for 24-36 hours, and the patients may require fluid therapy, but usually recover rapidly once convalescence starts. The hematological manifestations include an increase in hematocrit, thrombocytopenia, a prolonged bleeding time, and an increased prothrombin time. The temperature returns to normal when capillary leakage occurs. The occurrence of capillary leakage differentiates DHF from DF. When plasma leakage is so profound that shock occurs, it is also referred to as dengue shock syndrome (DSS).

\section{T-CELL RESPONSES TO DENV}

T-cells are known to play an important role in recovery from virus infections in general. Analysis of T-cell responses to DENV is essential to understand the mechanism of recovery from DENV infections and also the pathogenesis of DHF. There are not very satisfactory animal models to analyze T-cell responses to dengue virus and pathogenesis of DHF, although many groups have attempted to do so [2].
Analysis of human peripheral blood mononuclear cells (PBMC) is still the best way to elucidate the DENV-specific T-cell responses.

\section{4-1. Analysis of memory T-cells}

T-cell responses to DENV have been analyzed mainly using memory T-cells in PBMCs. DENV-specific memory CD4+CD8- T-cells and CD4-CD8+ T cells were detected in PBMCs from human subjects after natural infection with dengue virus, or after immunization with experimental, live-attenuated dengue vaccines.

\section{4-2. Analysis of DENV-specific CD4+ memory T-cells}

Specific CD4+CD8- memory T-cells induced by primary DENV infection were serotype-cross-reactive in bulk culture analysis, but the highest response was to the same serotype that caused the infection [3]. In some subjects, however, CD4+ T-cell memory responses after primary infection were serotype-specific [4]. The presence of serotype-cross-reactive and serotype-specific CD4+ T-cells was also demonstrated in precursor frequency assays [5]. In PBMC from DENV-1-immunizued individuals, the frequency of DENV-1 responsive T-cells was nearly 10 times higher than the frequency of other three serotypes [5].

DENV-specific CD4+ T-cell clones were established and analyzed for serotype specificities and functions. Most of the T-cell clones demonstrated specific cytotoxic activities. Consistent with the results of bulk culture analyses, DENVspecific CD4+ T-cell clones established from respective subjects have at least two types of serotype specificities: dengue serotype-specific and serotype-cross-reactive [6, 7]. The patterns of serotype cross-reactivities, and the proportion of serotype-specific and cross-reactive clones varied among individuals.

\section{4-3. Analyses of DENV-specific CD8+ memory T-cells}

DENV-specific CD8+CD4- memory T-cells induced by primary DENV infections were serotype cross-reactive in bulk culture analysis, and the highest response was to the same serotype that caused the primary infection [8]. DENVspecific CD8+ T-cell clones were established. The clones also contained serotype-specific and serotype cross-reactive clones [9]. Thus, similar to CD4+ T-cell responses, DENV infection with one serotype of DENV induces both serotypespecific and serotype cross-reactive CD8+ memory T-cells.

\section{PROTEINS RECOGNIZED BY DENV-SPECIFIC T-CELLS}

Proteins recognized by DENV-specific T-cells were determined in bulk culture and T-cell clonal analyses. In 
these analyses, the non-structural proteins NS3 and NS1,2a were determined to be the proteins mainly recognized by both CD4+T cells and CD8+T cells [10-13]. However, other proteins such as $\mathrm{C}, \mathrm{E}$ and NS4b also contained T cell epitopes [14-16]. The recognition pattern of the proteins differs according to the type of HLA.

\section{ACTIVATION OF T CELLS IN VIVO IN PATIENTS WITH DF/DHF}

Activation of DENV-specific T cells in vivo has been analyzed using PBMC samples obtained in early stages of DF and DHF by some groups. Green et al. reported that absolute counts of CD4+ T-cells, CD8+ T-cells, NK cells, and $\gamma \delta$ T-cells were decreased in children with DHF compared to those with DF early in the course of illness. The percentage of cells expressing CD69, an activation marker, was more increased in CD8+ T-cell and NK cell populations in children who developed DHF than in those with DF [17]. Mongkolsapaya et al. reported that many CD8+ T-cells possessed an activation phenotype and were undergoing programmed cell death [18]. These studies provided direct evidence of T-cell activation in vivo in the acute phase of DENV infections.

Activation of T-cells has also been suggested by increased levels of lymphokines in serum or plasma samples from patients with DF/DHF. It has been reported that levels of IL-2, IFN $\gamma$ and other lymphokines are highly elevated in patients with DHF compared to those with DF, and healthy subjects [19]. Serum levels of soluble tumor necrosis factor receptors (sTNFRs), soluble CD8 and soluble IL-2 receptor were higher in children who developed DHF than in those with DF or non-dengue febrile illness [20]. These studies indicated that T-cells are highly activated in patients with DF and DHF, and the levels of T cell activation are higher in patients with DHF than in those with DF. The results also suggested that activation of CD8+ T-cells is more profound than that of CD4+ T-cells. The results suggesting massive Tcell activation in DHF are summarized in Table 1.

\section{DETERMINATION OF THE CLONALITY OF T-CELLS ACTIVATED IN VIVO IN PATIENTS WITH DF/DHF}

As stated above, T-cells are highly activated in patients with DF and DHF, and there is a positive relationship between the levels of T-cell activation and severity of dengue illness [21]. One of the important questions is: Are the majority of activated T-cells DENV-specific, or are most of the activated T cells non-specific for DENV and activated as bystanders? This question was addressed by
Table 1. Characteristics of T-cell responses in patients with DF and DHF.

1. T-cell responses to DENV in primary infection are serotype cross-reactive, but the highest response is to the serotype that caused the primary infection. Most of the memory T-cells after secondary infection are serotype cross-reactive.

2. Massive T-cell activation in DHF.

* High percentage of CD69+ T-cells.

* The levels of T-cell activation: Higher in CD8+ T-cell poputation than in CD4+ T-cell population.

* High levels of lymphokines: IL-2, IL-6, IL-8, IL-10, TNF- $\alpha$, IFN- $\gamma$

* High levels of cellular proteins: sIL-2R, sCD4, sCD8, sTNFR

analyzing T-cell receptors.

The usage of T-cell receptor $\alpha$-chain variable region (TCRAV) and $\beta$-chain variable region (TCRBV) repertoires was analyzed in patients with DF or DHF. This analysis can determine whether T-cell activation in the patients is antigen-specific. If common antigens are responsible for proliferation of the T-cells, complementarity-determining region 3 (CDR3) diversity should be constrained, and homologous CDR3 sequences might be found in the T cells $[22,23]$. We then determined the nucleotide sequences of CDR3 of TCR $\alpha$-chain and $\beta$-chain gene from the T-cells. The delta score was used as an indicator to evaluate the extent of skew of TCR repertoires [24]. The delta score indicates the sum of absolute differences between the frequencies of respective $\mathrm{V}$ segments.

Peripheral blood mononuclear cells (PBMC) were collected from patients with DF or DHF on study days -4 , $-2,2$ and 180, where day 0 is defined as the day of defervescence. These donors developed dengue illness after secondary infections. TCR analysis was performed by microplate hybridization assay, size spectratyping and sequencing of CDR3 region. We determined whether T-cells are oligoclonally expanded in dengue patients, and whether there is a unique T-cell receptor usage in patients with DHF or DF.

The patterns of T-cell receptor usage changed in individuals during the course of illness. The pattern of TCR usage on day 180 was considered the control for each of the patients. Representative data for TCR VB usage in PBMC from a donor is shown in Figure 1. The percentages of $\mathrm{T}$ cells with VB051 and VB091 increased significantly. The TCRs that demonstrated an increase in percentage differ from donor to donor. In order to estimate the levels of skew in TCR usage, delta scores were calculated (Figure 2). The delta scores changed between acute and convalescent stages, suggesting the skew in TCR usage. There was a positive relationship between delta scores and the severity of dengue illness; the levels of skew of TCR usage were greater in 


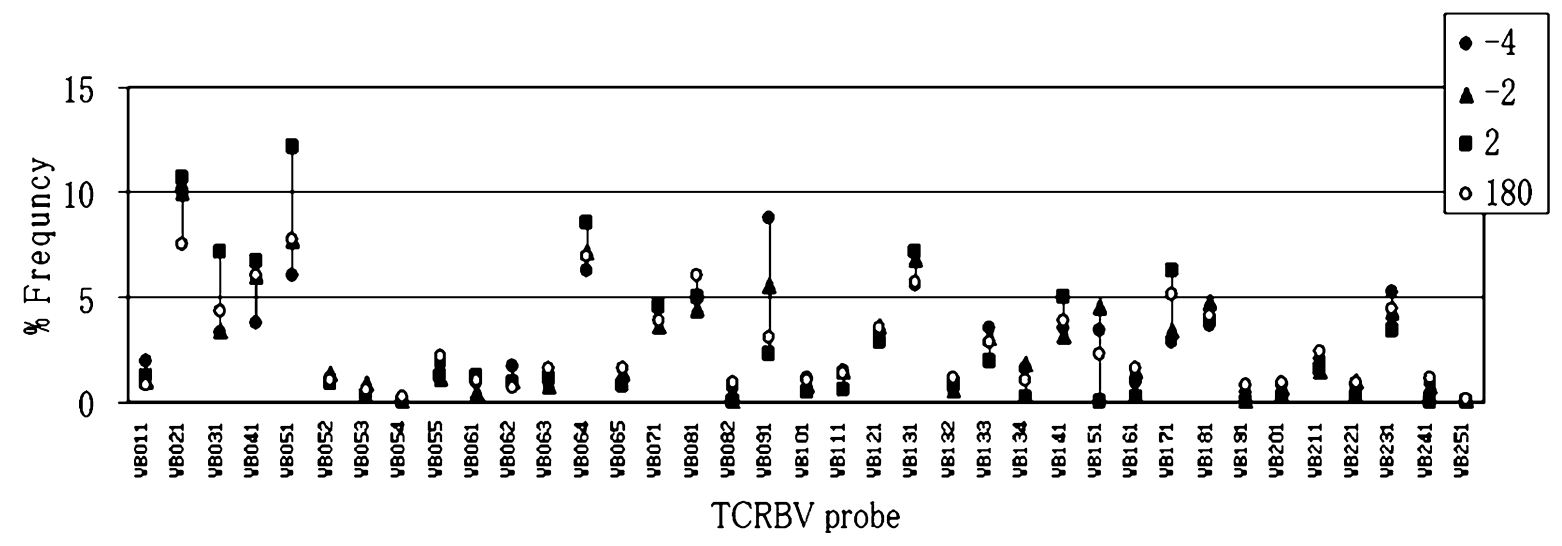

Fig. 1. T-cell receptor usage in PBMCs collected from a patient with DHF.

TCR VB usage in PBMC from a patient with DHF. PBMCs were collected on days $-4,-2,2$ and 180 , where day 0 is defined as the day of defervescence.
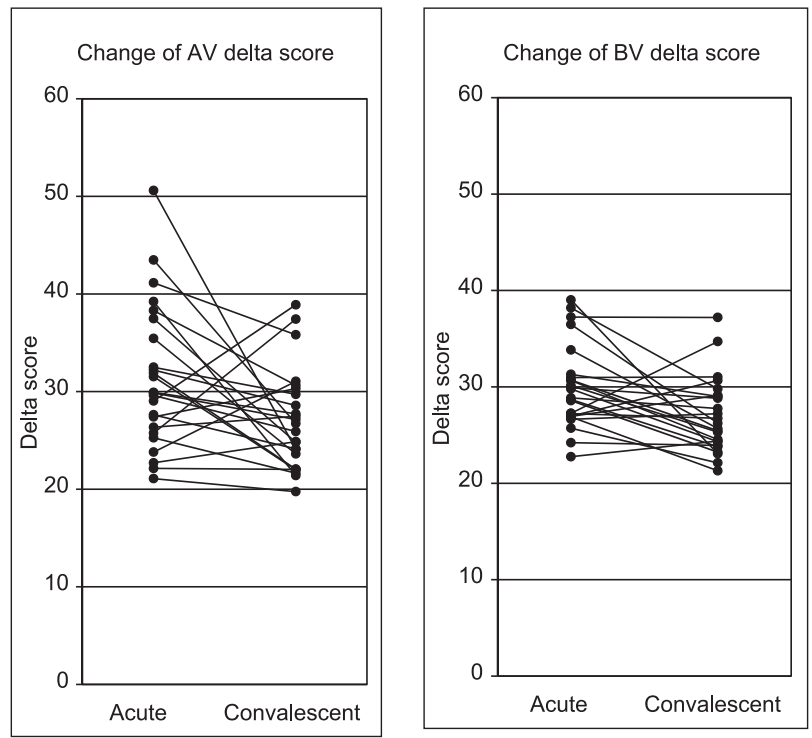

Fig. 2. Delta scores of TCR AV and BV.

Delta scores were compared between acute and convalescent stages. $\mathrm{P}<0.01$ between acute and convalescent stages in TCR AV and BV.

patients with DHF grades 3 and 4 (Figure 3). Clonality of activated T-cells was then evaluated by size spectratyping of the CDR3 of TCRs that demonstrated increased usage. The CDR3 of these TCRs showed only a few peaks, suggesting that T-cells with these TCRs proliferated in an oligoclonal fashion. The nucleotide sequences of the CDR3 regions of these TCRs showed that a high percentage of the
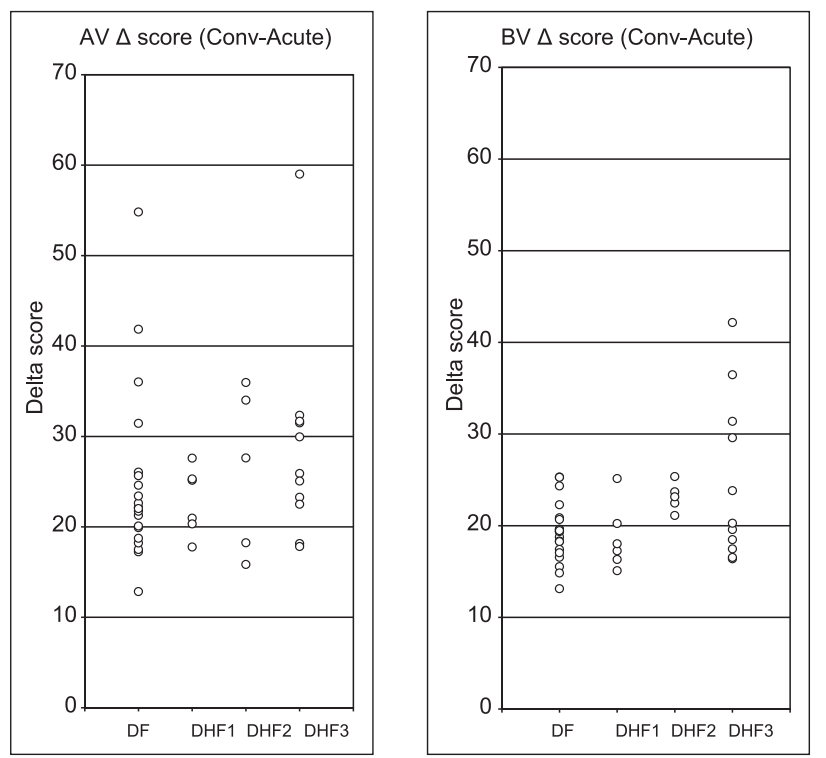

Fig. 3. Relationship between TCR delta scores and the severity of dengue illness.

There was a positive relationship between delta scores and severity of dengue illness in TCR BV usage.

sequenced CDR3 clones had identical amino acid sequences (Table 2). These results are consistent with those of the spectratyping analyses and confirmed that activation of Tcells is oligoclonal in some of the DHF patients (Figure 4). The results suggest that the majority of T-cells highly activated in vivo are DENV-specific in patients with DHF. 
Table 2. Alignment of amino acid sequences of CDR3 regions in TCRs that demonstrated increased levels of usage

\begin{tabular}{lccclclc}
\hline \multirow{2}{*}{ Patient } & \multirow{2}{*}{ Fever Days } & \multirow{2}{*}{ Occurrence } & \multirow{2}{*}{ AV/BV segment } & \multicolumn{3}{c}{ Amino acid sequences in CDR3 region } & \multirow{2}{*}{ AJ/BJ segment } \\
\cline { 5 - 7 } & & & AV/BV seq. & N (N-D-N) & AJ/BJ seq. & \\
K94-017 & 0 & $2 / 7$ & AV1S4 & YFCAVS & P & NRDDKIIFG & AJ30 \\
K94-029 & 1 & $3 / 8$ & AV1S4 & YFCAVS & AS & NSGGSNYKLTFG & AJ53 \\
K94-029 & 1 & $5 / 7$ & AV21S1 & YFCAAS & F & SGGYQKVTFG & AJ13 \\
K94-041 & $-4 \&-2$ & $6 / 7$ & AV31S1 & YLCA & AEY & TGGFKTIFG & AJ9 \\
K94-007 & 1 & $3 / 11$ & AV14S1 & YFCAYRS & GG & YKLSFG & AJ20 \\
C94-138 & 0 & $5 / 7$ & AV20S1 & YYCLVGD & D & DYKLSFG & AJ20 \\
K94-041 & -4 & $6 / 6$ & BV9S1 & YFCASS & FS & NEQFFG & BJ2S1 \\
& -2 & $3 / 5$ & BV9S1 & YFCASSQ & DRD & SGANVLTFG & BJ2S6 \\
K94-004 & -1 & $8 / 11$ & BV6S5 & YLCASS & RGG & EQYFG & BJ2S7 \\
C94-081 & -2 & $2 / 7$ & BV8S1 & YFCASS & YG & NEQFFG & BJ2S1 \\
\hline
\end{tabular}

\section{K94-041}

VA072

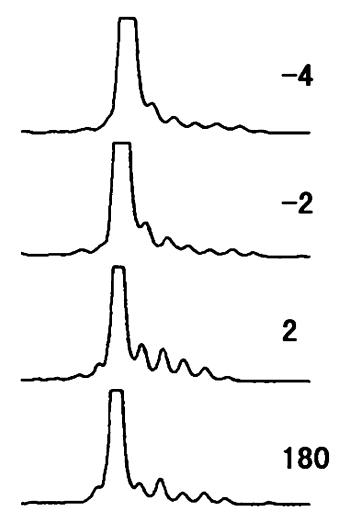

K94-041

VA211
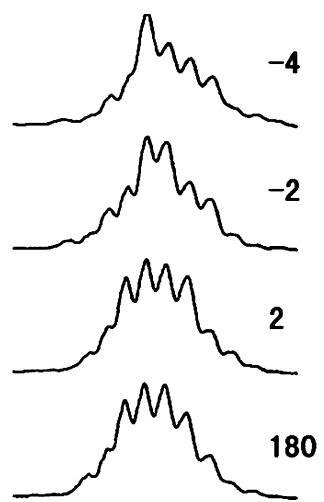

K94-041

VA311

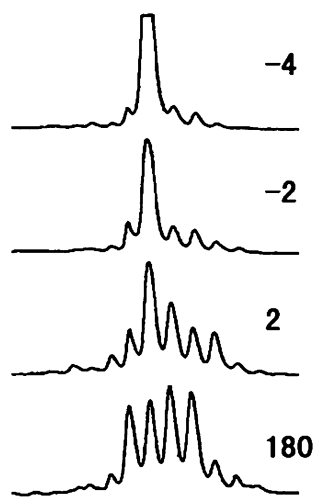

C94-066

VB021

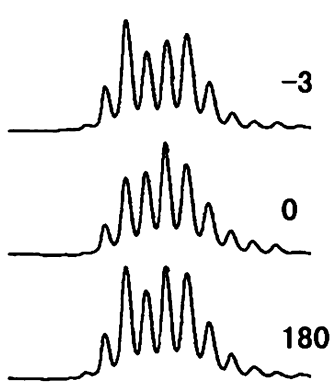

K94-004

VB064

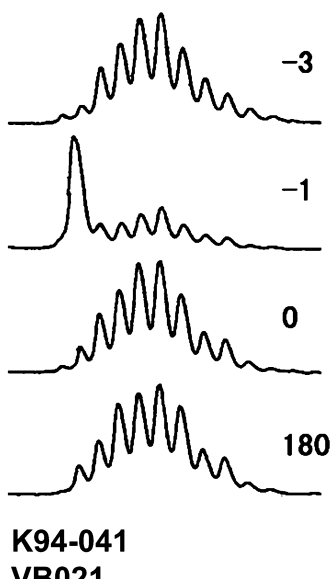

VB021

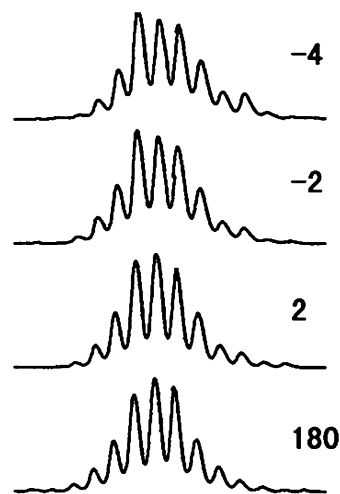

K94-041

VB051

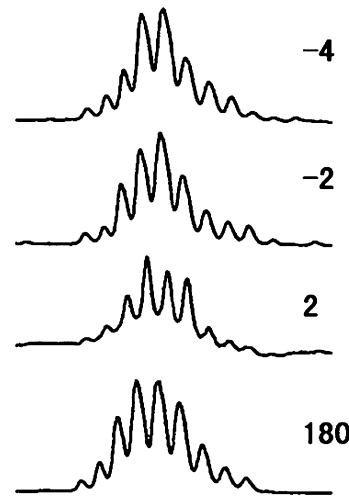

K94-041

VB091

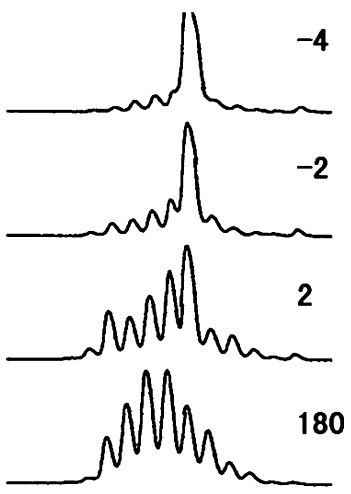

Fig. 4. Size spectratyping of CDR3 of selected TCRs in PBMCs obtained from patients with DF and DHF. PBMCs were collected on days $-4,-2,2$ and 180 , where day 0 is defined as the day of defervescence.

\section{ROLE OF T-CELLS IN THE PATHOGENESIS OF DHF}

The role of the activated T-cells in the pathogenesis of DHF has been hypothesized [25]. A series of studies have suggested that plasma leakage, which differentiates DHF from DF, is caused by malfunction of vascular endothelial cells induced by cytokines or chemical mediators rather than by destruction of the small vessels [26, 27]. Plasma levels of various lymphokines are significantly higher in DHF than in DF. The lymphokines elevated in patients with 
DHF include TNF- $\alpha$, IL-2, IL-6, IL-8, IL-10, IL-12 and IFN- $\gamma$. However, it is not clearly understood how these lymphokines cause malfunction of vascular endothelial cells and lead to plasma leakage.

Some of the cytokines, i.e. IFN- $\gamma$, IL- 2 and TNF- $\alpha$ were mainly produced by DENV-specific T-cells upon activation [28]. It is likely, therefore, that activated specific T-cells are responsible for increased levels of cytokines that play a key role in the pathogenesis of DHF. Consistent with the assumption, there was a correlation between the levels T-cell activation and dengue disease severity. It should, however, be understood that these activated T-cells may also contribute to the recovery from DENV infection, as has been reported in other virus infections.

\section{CONCLUSIONS}

New findings regarding T-cell responses to DENV have been accumulating. However, a complete understanding of the T-cell activation in patients with DF and DHF and the roles of activated T-cells in recovery and in the pathogenesis of DHF remains a challenge. A better understanding of T-cell responses as well as function and role in DENV infection will facilitate the development of dengue vaccines, new treatment programs and measures to prevent DENV infection.

\section{REFERENCES}

1. World Health Organization. Dengue: Guidelines for diagnosis, treatment, prevention and control. Geneva: WHO; 2009. pp. 25-58.

2. Omatsu T, Moi ML, Hirayama T, Takasaki T, Nakamura S, Tajima S, Ito M, Yoshida T, Saito A, Katakai Y, Akari $\mathrm{H}$, Kurane I. Common marmoset (Callithrix jacchus) as a primate model of dengue virus infection: development of high levels of viremia and demonstration of protective immunity. J Gen Virol 2011; 92: 2272-2280.

3. Kurane I, Innis BL, Nisalak A, Hoke C, Nammannitya S, Meager A, Ennis FA. Human T cell responses to dengue virus antigens: proliferative responses and interferon gamma production. J Clin Invest 1989; 83: 506-513.

4. Dharakul T, Kurane I, Bhamarapravati N, Suttee Y, Vaughn DW, Hoke CH, Ennis FA. Dengue virus-specific memory $\mathrm{T}$ cell responses in human volunteers receiving a live attenuated dengue- 2 virus candidate vaccine. J Infect Dis 1994; 170: 27-33.

5. Green S, Kurane I, Tacket CO, Edelman R, Eckels KH, Vaughn DW, Hoke CH, Ennis FA. Dengue virus-specific human $\mathrm{CD} 4+\mathrm{T}$-lymphocyte responses in a recipient of an experimental live-attenuated dengue virus type 1 vaccine: bulk culture proliferation, clonal analysis, and precursor frequency determination. J Virol 1993; 67: 5962-5967.

6. Kurane I, Meager A, Ennis FA. Dengue virus-specific human T cell clones: Serotype crossreactive proliferation, interferon gamma production, and cytotoxic activity. J Exp Med 1989; 170: 763-775.

7. Kurane I, Brinton MA, Samson AL, Ennis FA. Dengue virus-specific, human CD4+ CD8- cytotoxic T-cell clones: multiple patterns of virus cross-reactivity recognized by NS3-specific T-cell clones. J Virol 1991; 65: 1823-1828.

8. Bukowski JF, Kurane I, Lai C-J, Bray M, Falgout B, Ennis FA. Dengue virus-specific cross-reactive CD8+ human cytotoxic T Iymphocytes. J Virol 1989; 63: 5086-5091.

9. Livingston PG, Kurane I, Dai LC, Okamoto Y, Lai CJ, Men R, Karaki S, Takiguchi M, Ennis FA. Dengue virusspecific, HLA-B35-restricted, human CD8+ cytotoxic T lymphocyte (CTL) clones. Recognition of NS3 amino acids 500 to 508 by CTL clones of two different serotype specificities. J Immunol 1995; 154: 1287-1295.

10. Kurane I, Dai L-C, Livingston P, Reed E, Ennis FA. Definition of an HLA-DPw2-restricted epitope on NS3, recognized by a dengue virus serotype-cross-reactive human CD4+ CD8- cytotoxic T-cell clone. J Virol 1993; 67: 62856288.

11. Mathew A, Kurane I, Rothman A, Zeng LL, Brinton MA, Ennis FA. Dominant recognition by human CD8+ cytotoxic T Iymphocytes (CTL) of dengue virus nostructural proteins NS3 and NS1,2a. J Clin Invest 1996; 98: 1684-1692.

12. Green S, Kurane I, Pincus S, Paoletti E, Ennis FA. Recognition of dengue virus NS1-NS2a proteins by human CD4+ cytotoxic T lymphocyte clones. Virology 1997; 234: 383386.

13. Mathew A, Kurane I, Green S, Stephens HAF, Vaughn DW, Kalayanarooj S, Suntayakorn S, Ennis FA, Rothman A. Predominance of CTL responses to serotype crossreactive epitopes on nonstructural protein following natural secondary dengue virus infection. J Virol 1998; 72: 3999_ 4004.

14. Gagnon SJ, Zeng WL, Kurane I, Ennis FA. Identification of two epitopes on the dengue 4 virus capsid protein recognized by a serotype-specific and a panel of serotypecross-reactive human CD4+ cytotoxic T-lymphocyte clones. J Virol 1996; 70: 141-147.

15. Livingston PC, Kurane I, Lai C-J, Bray M, Ennis FA. Recognition of envelope protein by dengue virus serotypespecific human CD4+CD8- cytotoxic T-cell clones. J Virol 1994; 68: 3283-3288.

16. Bashyam HS, Green S, Rothman AL. Dengue virus-reactive CD8+ T cells display quantitative and qualitative differences in their response to variant epitopes of heterologous viral serotypes. J Immunol 2006; 176: 2817-2824.

17. Green S, Pichyangkul S, Vaughn DW, Kalayanarooj S, Nimmannitya S, Nisalak A, Kurane I, Rothman AL, Ennis FA. Early CD69 expression on peripheral blood lymphocytes from children with dengue hemorrhagic fever. J Infect Dis 1999; 180: 1429-1435.

18. Mongkolsapaya J, Dejnirattisai W, Xu XN, Vasanawathana S, Tangthawornchaikul N, Chairunsri A, Sawasdivorn S, Duangchinda T, Dong T, Rowland-Jones S, Yenchitsomanus PT, McMichael A, Malasit P, Screaton G. Original anti- 
genic sin and apoptosis in the pathogenesis of dengue hemorrhagic fever. Nat Med 2003; 9: 921-927.

19. Kurane I, Innis BL, Nimmannitya S, Nisalak A, Meager A, Janus J, Ennis FA. Activation of T lymphocytes in dengue virus infections. High levels of soluble interleukin 2 receptor, soluble $\mathrm{CD} 4$, soluble $\mathrm{CD} 8$, interleukin 2 , and interferon-gamma in sera of children with dengue. J Clin Invest 1991; 88: 1473-1480.

20. Green S, Vaughn DW, Kalayanarooj S, Nimmannitya S, Suntayakorn S, Nisalak A, Lew R, Innis BL, Kurane I, Rothman AL, Ennis FA. Early immune activation in acute dengue illness is related to development of plasma leakage and disease severity. J Infect Dis 1999; 179: 755-762.

21. Zivna I, Green S, Vaughn DW, Kalayanarooj S, Stephens HA, Chandanayingyong D, Nisalak A, Ennis FA, Rothman AL. T cell responses to an HLA-B*07-restricted epitope on the dengue NS3 protein correlate with disease severity. J Immunol 2002; 168: 5959-5965.

22. Hirokawa M, Horiuchi T, Kawabata Y, Kitabayashi A, Saitoh H, Ichikawa Y, Matsutani T, Yoshioka T, Tsuruta Y, Suzuki R, Miura A. Oligoclonal expansion of CD4(+)CD28(-) T lymphocytes in recipients of allogeneic hematopoietic cell grafts and identification of the same $\mathrm{T}$ cell clones within both CD4(+)CD28(+) and CD4(+)CD28(-) T cell subsets. Bone Marrow Transplant
2001; 27: 1095-1100.

23. Matsutani T, Shiiba K, Yoshioka T, Tsuruta Y, Suzuki R, Ochi T, Itoh T, Musha H, Mizoi T, Sasaki I. Evidence for existence of oligoclonal tumor-infiltrating lymphocytes and predominant production of $\mathrm{T}$ helper $1 / \mathrm{T}$ cytotoxic 1 type cytokines in gastric and colorectal tumors. Int J Oncol 2004; 25: 133-141.

24. Matsutani T, Ohashi Y, Yoshioka T, Tsuruta Y, Doi H, Satomi S, Suzuki R. Skew in T-cell receptor usage and clonal T-cell expansion in patients with chronic rejection of transplanted kidneys. Transplantation 2003; 75: 398407.

25. Kurane I. Dengue hemorrhagic fever with special emphasis on immunopathogenesis. Comp Immunol Microbiol Infect Dis 2007; 30(5-6): 329-340.

26. Green $S$, Rothman A. Immunopathological mechanisms in dengue and dengue hemorrhagic fever. Curr Opin Infect Dis 2006; 19: 429-436.

27. Kurane I, Ennis FE. Immunity and immunopathology in dengue virus infections. Semin Immunol 1992; 4: 121-127.

28. Gagnon SJ, Ennis FA, Rothman AL. Bystander target cell lysis and cytokine production by dengue virus-specific human $\mathrm{CD} 4(+)$ cytotoxic T-lymphocyte clones. J Virol 1999; 73: 3623-3629. 